International Journal of Multidisciplinary Research AND ANALysis

ISSN(print): 2643-9840, ISSN(online): 2643-9875

Volume 04 Issue 10 October 2021

DOI: 10.47191/ijmra/v4-i10-13, Impact Factor: 6.072

Page No.- 1426-1430

\title{
Interval Version of the Operational Calculation Method for Solving Ordinary Differential Equations
}

\author{
Oybek Zhumaboyevich Khudayberdiyev \\ Navoi State Mining Institute Navoiy, Republic of Uzbekistan
}

ABSTRACT: This article discusses the interval variant of solving ordinary differential equations with given initial conditions, i.e. the Cauchy problem, by the method of operational calculus. This is where the interval version of the operational calculus is motivated and built. As a result, on the basis of the proved theorem in this article, an analytic interval set of solutions is obtained that is guaranteed to contain a real solution to the problem.

KEYWORDS: Ordinary differential equation, real solution, interval, interval solution, interval arithmetic, full Caucher interval arithmetic, extension function, width function, interval-valued function, original, image, Laplace transform, operational calculus method.

\section{INTRODUCTION}

When solving most technical problems, its mathematical model is reduced to differential equations. As is known, the use of interval methods allows one to obtain guaranteed two-sided estimates for solutions of differential equations, but the practical calculation of these estimates is associated with the problem of exponential mutual divergence of the found boundaries - a phenomenon that received the name of the wrapping effect (in the English-language literature - the wrapping effect, and in Russian works the names often appear - the wrapping effect or the Moore effect) [1, 2]. This phenomenon manifests itself in one way or another in most interval and two-sided methods for evaluating solutions of ordinary differential equations and is explained by several, often overlapping, reasons [2].

The proposed method of operational calculus is motivated by the fact that when using this method to solve ordinary differential equations (ODE) with given initial conditions (Cauchy problems), only four arithmetic operations and a ready-to-use table of transition from one type of function to another and back are applied. The interval version of this method expands the possibilities of finding a solution to the Cauchy problem with an acceptable width of the solution intervals. Further, we briefly outline the theory of operational calculus, since the facts presented will be used to describe the real and interval versions of this method.

\section{THE MAIN FINDINGS AND RESULTS}

Let the function $f(t)$ satisfy the following conditions [3]:

1. $f(t)=0$ for all $t<0$;

2. $f(\mathrm{t})$ piecewise continuous function;

3. $\exists M>0$ and $\forall s_{0}>0$ such that $|f(\mathrm{t})| \leq \operatorname{Mexp}\left(s_{0} t\right)$.

Then the function $f(t)$ is called the original (initial function). We multiply the function $f(t)$ by the complex-valued function exp($p t)(\operatorname{Re} p>0)$, then we obtain $f(t) \exp (-p t)$ and integrate this function within the limits $[0 ; \infty]$ in the variable $t$ :

$$
\int_{0}^{\infty} f(t) e^{-p t} d t
$$

Improper integral (1) exists due to conditions 1) -3) and is a function of the parameter $\mathrm{p}$ [3]. We denote it by $F(p)$. Then we get

$$
F(p)=\int_{0}^{\infty} f(t) e^{-p t} d t
$$

The function $F(p)$ is called the image or the Laplace transform of the function $f(\mathrm{t})$ and is denoted by $L\{\mathrm{f}(\mathrm{t})\}=F(p)$.

The function $F(p)$ satisfies the properties of linearity, shift, displacement, etc. [3]. Transition tables from one function to another are given in many literatures on higher mathematics, for example, in $[3,5]$. 


\section{Interval Version of the Operational Calculation Method for Solving Ordinary Differential Equations}

Since the integral (1) exists and its value is equal to $F(p)$ [3], then the further discussion will be considered justified.

As you know, from the course of higher mathematics, methods of operational calculus are used to solve ODEs or systems of ODEs with initial conditions, i.e. for solutions of the Cauchy problem.

Next, we will briefly outline the real version of the statement of the Cauchy problem and its solution by the method of operational calculus.

\section{REAL VARIANT OF THE METHOD OF OPERATIONAL CALCULUS}

Let an $n$-th order ODE with constant coefficients be given:

$$
y^{(n)}+a_{1} y^{(n-1)}+a_{2} y^{(n-2)}+\cdots+a_{n} y=f(t) .
$$

It is required to find a particular solution of equation (3) that satisfies the initial conditions

$$
y(0)=y_{0}, y^{\prime}(0)=y_{1}, y^{\prime \prime}(0)=y_{2}, \ldots, y^{(n-1)}(0)=y_{n-1},
$$

where $y_{0}, y_{1}, \ldots, y_{n-1}$ are given constant numbers.

We will assume that the sought-for function y $(t)$ together with its derivatives up to the $n$-th order inclusive and the function $f(\mathrm{t})$ are originals [3].

Now, using the properties of linearity and differentiation of the original, taking into account the initial conditions (4), we find the image of both sides of equation (3), then we get

$$
\begin{gathered}
\left(p^{n} Y(p)-p^{n-1} y_{0}-p^{n-2} y_{1}-\cdots-y_{n-1}\right)+a_{1}\left(p^{n-1} Y(p)-p^{n-2} y_{0}-p^{n-3} y_{1}-\cdots-y_{n-2}\right)+\cdots a_{n-1}\left(p Y(p)-y_{0}\right)+ \\
a_{n} Y(p)=F(p)
\end{gathered}
$$

This equation is called the operator equation or the equation in images. Equation (5) can be rewritten as follows:

$$
\begin{gathered}
Y(p)\left[p^{n}+a_{1} p^{n-1}+a_{2} p^{n-2}+\cdots+a_{n-1} p+a_{n}\right]=F(p)+y_{0}\left(p^{n-1}++a_{1} p^{n-2}+a_{2} p^{n-3}+\cdots+a_{n-1}\right)+y_{1}\left(p^{n-2}+\right. \\
\left.a_{1} p^{n-3}+a_{2} p^{n-4}+\cdots++a_{n-2}\right)+\cdots y_{n-1} .
\end{gathered}
$$

For brevity, we denote

$$
\begin{gathered}
Q_{n}(p)=p^{n}+a_{1} p^{n-1}+a_{2} p^{n-2}+\cdots+a_{n-1} p+a_{n}, \\
R_{n-1}(p)=y_{0}\left(p^{n-1}+a_{1} p^{n-2}+a_{2} p^{n-3}+\cdots+a_{n-1}\right)+ \\
+y_{1}\left(p^{n-2}+a_{1} p^{n-3}+a_{2} p^{n-4}+\cdots+a_{n-2}\right)+\cdots y_{n-1} .
\end{gathered}
$$

Here $Q_{n}(p)$ is the $n$-th degree polynomial, $R_{n-1}(p)$ is the $(n-1)$ th degree polynomial with respect to the parameter $p$. Then equation (6) can be written as follow

$$
Y(p) Q_{n}(p)=F(p)+R_{n-1}(p)
$$

or

$$
Y(p)=\frac{F(p)+R_{n-1}(p)}{Q_{n}(p)} .
$$

Equation (7) is called the operator solution of this differential equation (3) with initial conditions (4).

If all initial conditions (4) are equal to zero, i.e.

$$
y_{0}=y_{1}=y_{2}=\cdots y_{n-1}=0, \text { then } R_{n-1}(p)=0,
$$

then equation (7) takes a simpler form:

$$
Y(p)=\frac{F(p)}{Q_{n}(p)} .
$$

Further, a rational fraction, i.e. the right-hand side of equality (7) (or (8)), expanding into simple fractions and passing to the originals, we find the required solution.

Now we turn to the presentation of the interval version of the method of operational calculus.

\section{INTERVAL VERSION OF THE OPERATIONAL CALCULUS METHOD}

To obtain interval generalized and/or natural extensions of one or another real function, we use the definitions given in [1, 2].

Comment. Henceforth, in accordance with [2]:

1) we will use the complete interval arithmetic of Kaucher, which is usually denoted by $\boldsymbol{K} \boldsymbol{R}$ in contrast to the space $\boldsymbol{I R}$. This means that if $* \in\{+,-, \cdot /\}$, then operations $\left({ }^{*}\right)$ are performed in $\boldsymbol{K} \boldsymbol{R}$.

2) Notation for variables and functions with the font "bold" means the interval of these values.

Note that through $\boldsymbol{t}=[\underline{t} ; \bar{t}]$ and $\boldsymbol{p}=[\underline{p} ; \bar{p}]$ denote intervals, and $\boldsymbol{Y}[t], \overline{\boldsymbol{Y}}[\boldsymbol{p}], \boldsymbol{F}(\boldsymbol{t})$ and $\overline{\boldsymbol{F}}(\boldsymbol{p})$ are interval-valued functions

To avoid ambiguity, we introduce the following designations, according to $[1,2]$ :

$f(t)$-original, $\mathrm{F}(\mathrm{p})$-image of function $f(t)$;

$\boldsymbol{F}(\boldsymbol{t})$-is a natural extension of the function $f(t)$; 


\section{Interval Version of the Operational Calculation Method for Solving Ordinary Differential Equations}

$\bar{F}(\boldsymbol{p})$ is a natural extension of the function $F(p)$;

$y(t)$ is the sought solution, $Y(p)$ is the image of the function $y(t)$;

$\boldsymbol{Y}[t]$ is a natural extension of the function $y(t)$;

$\overline{\boldsymbol{Y}}[\boldsymbol{p}]$ is a natural extension of the function $Y(p)$,

where $p=a+i b$ is a complex number, $\boldsymbol{p}=\boldsymbol{a}+i \boldsymbol{b}$ is a complex interval of the form $\boldsymbol{p}=[p, \bar{p}]$.

Using the above notation, we describe the interval version of the Cauchy problem (3) with initial conditions (4).

Let the function $y(t)$ have an interval extension $\boldsymbol{Y}[\boldsymbol{t}]$, that is $y(t) \in \boldsymbol{Y}[t]$, with the following properties [1]:

1) $\boldsymbol{Y}[\boldsymbol{t}]$ is defined and continuous on the interval $\boldsymbol{A}=[a, b]$;

2) $\boldsymbol{Y}[\boldsymbol{t}]$ is monotonic with respect to inclusion.

Let an ODE of the nth order with constant coefficients be given

$$
\boldsymbol{Y}^{(n)}+a_{1} \boldsymbol{Y}^{(n-1)}+a_{2} \boldsymbol{Y}^{(n-2)}+\cdots+a_{n} \boldsymbol{Y}=\boldsymbol{F}[\mathrm{t}],
$$

and initial conditions

$$
\boldsymbol{Y}[0]=\boldsymbol{Y}_{0}, \boldsymbol{Y}^{\prime}[0]=\boldsymbol{Y}_{1}, \boldsymbol{Y}^{\prime \prime}[0]=\boldsymbol{Y}_{2}, \ldots, \boldsymbol{Y}^{(n-1)}[0]=\boldsymbol{Y}_{n-1} .
$$

It is required to find solutions $y(t) \in Y[t]$, where the initial conditions, which are contained in the corresponding intervals

$$
y_{0} \in \boldsymbol{Y}_{0}, y_{1} \in \boldsymbol{Y}_{1}, \ldots y_{n-1} \in \boldsymbol{Y}_{n-1} \text {, }
$$

where $\boldsymbol{Y}_{0}, \boldsymbol{Y}_{1}, \ldots, \boldsymbol{Y}_{n-1}$-intervals. Those. we will seek a particular solution of equation (9) with initial conditions (10) satisfying the inclusion

$$
y(t) \in \boldsymbol{Y}[t] \text {. }
$$

To solve the problem, the following theorem is required.

Theorem. If $\boldsymbol{Y}[\boldsymbol{t}]$ is continuous and monotone with respect to inclusion, and also for $\forall \boldsymbol{t}_{0} \subset \boldsymbol{t}=[\underline{t}, \bar{t}]$ the conditions $w\left(\boldsymbol{Y}[t]-\boldsymbol{Y}_{0}\right) \leq l_{1} w\left(\boldsymbol{t}-t_{\mathbf{0}}\right) u w\left(\boldsymbol{Y}_{0}\right) \leq l_{2} w\left(\boldsymbol{t}_{0}\right)$,

then

1) the interval set of solutions $\boldsymbol{Y}[t]$ contains the desired real solution y $(\mathrm{t})$ on some interval $\boldsymbol{A}=[\mathrm{a}, \mathrm{b}]$;

2) there is a number $1>0$ such that $w(\boldsymbol{Y}[\boldsymbol{t}]) \leq \operatorname{lw}(\boldsymbol{t})$ for all $\boldsymbol{t} \in(a, b)$.

Evidence. The proof of the first part follows from property 1) -2) of the function $\boldsymbol{Y}[t]$. Let us prove the second part of the theorem. For this we use the properties of the width function wid.

$$
\begin{gathered}
w(\boldsymbol{Y}[t])=w\left(\boldsymbol{Y}[t]-\boldsymbol{Y}_{0}+\boldsymbol{Y}_{0}\right) \leq w\left(\boldsymbol{Y}[t]-\boldsymbol{Y}_{0}\right)+w\left(\boldsymbol{Y}_{0}\right) \leq \\
\leq l_{1} w\left(\boldsymbol{t}-\boldsymbol{t}_{\mathbf{0}}\right)+l_{2} w\left(\boldsymbol{t}_{\mathbf{0}}\right) \leq l_{1} w(\boldsymbol{t})+l_{2} w\left(\boldsymbol{t}_{\mathbf{0}}\right) \leq \\
\leq l_{1} w(\boldsymbol{t})+l_{2} w(\boldsymbol{t}) \leq\left(l_{1}+l_{2}\right) w(\boldsymbol{t})=l w(\boldsymbol{t}) .
\end{gathered}
$$

hus, we have $w(\boldsymbol{Y}[t]) \leq l w(\boldsymbol{t})$, where $l=l_{1}+l_{2}$ is a constant and does not depend on $\mathrm{t}$. The theorem is proved. Now, using the introduced notation, we write the interval version of the operator solutions (7) and (8):

$$
\overline{\boldsymbol{Y}}[\boldsymbol{p}]=\frac{\overline{\boldsymbol{F}}[\boldsymbol{p}]+\overline{\boldsymbol{R}}_{n-1}[\boldsymbol{p}]}{\overline{\boldsymbol{Q}}_{n}[\boldsymbol{p}]},
$$

or, if all initial conditions are equal to zero, i.e. . $\overline{\boldsymbol{R}}_{n-1}[\boldsymbol{p}]=0$ then

$$
\bar{Y}[\boldsymbol{p}]=\frac{\overline{\boldsymbol{F}}[\boldsymbol{p}]}{\overline{\boldsymbol{Q}}_{n}[\boldsymbol{p}]} \text {. }
$$

Expanding the rational fraction, i.e. the right-hand side of equality (13) (or (14)), into the simplest fractions, we obtain images of functions in tabular form or functions that lead to them. Further, passing from the images to the initial functions, we obtain the interval solution $\boldsymbol{Y}[t]$ of the problem. By the proved theorem, the desired solution is $y(t) \in \boldsymbol{Y}[t]$.

We give from [5] an important statement, the use of which, when finding the originals, is very effective.

If the image $F(p)=\frac{A(p)}{B(p)}$ is a regular rational fraction, the denominator $B(p)$ has only simple roots $p_{1}, p_{2}, \ldots, p_{n}$ then the function

$$
f(t)=\sum_{k=1}^{n} \frac{A\left(p_{k}\right)}{B^{\prime}\left(p_{k}\right)} e^{k t}
$$

is the original with the image $F(p)$.

In our case, this statement refers to the function

$$
\overline{\boldsymbol{Y}}[\boldsymbol{p}]=\frac{\overline{\boldsymbol{F}}[\boldsymbol{p}]}{\overline{\boldsymbol{Q}}_{n}[\boldsymbol{p}]} .
$$

Now let's consider an example for the application of the above operating method in an interval version.

The main forms of representation of finite-dimensional linear continuous stationary operators of transformation of input variables $f(t)$ into output variables y $(t)$ are: differential equations, transfer functions, etc. For one-dimensional systems, the variables $f(t)$ and $y(t)$ are scalars. These and some other representations of the operators of the considered class of models 


\section{Interval Version of the Operational Calculation Method for Solving Ordinary Differential Equations}

can be taken as the basis for setting dynamic properties in terms of input-output. If one form or another turns out to be more preferable for specific studies, the problem of transition from one form to another is posed and solved, for example, the problem of constructing time and frequency characteristics from a differential equation or transfer function. The simplest formal transition is by replacing the differentiation operator with a complex argument from differential equation (3) to transfer function (7) and vice versa. The transfer function is equal to the ratio of the Laplace images of the output and input variables at zero initial conditions [4]:

$$
W(p)=Y(p) / F(p)
$$

Knowing the transfer function and the variable input image, it is easy to find the output image

$$
Y(p)=W(p) F(p)
$$

An example from [4], studied in the real case of the "input-output" model, will be considered in the interval version. Example. Let the system be described by a second-order differential equation:

$$
a_{2} \frac{d^{2} y}{d t^{2}}+a_{1} \frac{d y}{d t}+a_{0} y=b_{0} f ; \quad y(0), \quad y^{\prime}(0) \text {. }
$$

Omitting, for brevity, intermediate calculations, we write the final form of the transfer function. For zero initial conditions $y(0)=y^{\prime}(0)=0$, the ratio of images, i.e. the transfer function has the following form

$$
W(p)=\frac{b_{0}}{a_{2} p^{2}+a_{1} p+a_{0}} .
$$

Let's put, for simplicity, $b_{0}=2, a_{2}=1, a_{1}=3, a_{0}=2$. Then the transfer function will be written in the form [4]:

$$
W(p)=\frac{2}{p^{2}+3 p+2} \text {. }
$$

If we take into account the fact that the input parameters can fluctuate in a certain range, and as a result, the output parameters can also be in a certain interval, then they can be described as interval values. For this reason, adhering to the above introduced notation, we rewrite equalities (18), (19) and (20) in interval form. Then we have

$$
\begin{gathered}
a_{2} \frac{d^{2} \boldsymbol{Y}}{d \boldsymbol{t}^{2}}+a_{1} \frac{d \boldsymbol{Y}}{d \boldsymbol{t}}+a_{0} \boldsymbol{Y}=b_{0} \boldsymbol{F}, \quad \boldsymbol{Y}(0)=\boldsymbol{Y}^{\prime}(0)=0, \\
W(\boldsymbol{p})=\frac{b_{0}}{a_{2} \boldsymbol{p}^{2}+a_{1} \boldsymbol{p}+a_{0}}, \\
W(\boldsymbol{p})=\frac{2}{\boldsymbol{p}^{2}+3 \boldsymbol{p}+2} .
\end{gathered}
$$

We expand function (23) into the simplest fractions

$$
W(\boldsymbol{p})=\frac{2}{\boldsymbol{p}^{2}+3 \boldsymbol{p}+2}=\frac{2}{\boldsymbol{p}+1}-\frac{2}{\boldsymbol{p}+2} .
$$

Now, using the inverse Laplace transform, we find the original for the right-hand side of equality (24:

$$
\boldsymbol{Y}[t]=2 e^{-t}-2 e^{-2 t} \text {. }
$$

Function (25) is the interval set of solutions to this differential equation (21).

The real solution of the differential equation (21), according to [4], has the following form

$$
y(t)=2 e^{-t}-2 e^{-2 t}
$$

Based on the theorem proved above, the interval set of solutions $\boldsymbol{Y}[t]$ (25) contains the obtained real solution $y(t)$ (26), since $t \in \boldsymbol{t}=[\underline{t} ; \bar{t}]$, i.e. $y(t) \in \boldsymbol{Y}[t]$.

This means that the interval set of solutions $Y$ [t] obtained in the integer version of the operational calculus method, based on the proved theorem, is guaranteed to contain the desired real solution to the Cauchy problem and thus obtain an analytical solution to the problem.

\section{CONCLUSION}

Thus, the obtained interval version of the solution of the ODE by the method of operational calculus, by virtue of the proved theorem, makes it possible to find an analytical set of solutions to these equations, which is guaranteed to contain a real solution to the problem posed and thereby successfully bypass the Moore effect.

\section{REFERENCES}

1) Kalmykov S.A., Shokin Yu.I., Yuldashev Z.Kh. Interval analysis methods. Novosibirsk, Science: 1986.-186p.

2) Shary S.P. Finite-dimensional interval analysis. Novosibirsk, Publishing house "XYZ" -2019. -632 p.

3) Piskunov N. P. Differential and integral calculus. Volume 2: Textbook for technical colleges. Moscow: Nauka-2008.-560s. 
Interval Version of the Operational Calculation Method for Solving Ordinary Differential Equations

4) B.M.Borisov, V.E.Bol'shakov, V.I.Malarev, R.M. Proskuryakov. Mathematical modeling and calculation of control systems for technical objects. Tutorial. SPb GGI, SPb, $2002.63 \mathrm{p}$.

5) D.T.Pismenniy. Lecture notes on higher mathematics. Part 2. $-\mathrm{M}$.: Rolf, 2000.-256 p. 